\title{
Antibiotics for Aspiration Pneumonia in Neurologically Impaired Children
}

\author{
Joanna Thomson, MD, MPH ${ }^{1,2 *}$, Matt Hall, $\mathrm{PhD}^{3}$, Lilliam Ambroggio, PhD, $\mathrm{MPH}^{4}$, Jay G Berry, MD, MPH5,6, \\ Bryan Stone, MD, MS ${ }^{7,8}$, Rajendu Srivastava, MD, MPH7,9, Samir S Shah, MD, MSCE ${ }^{1,2,10}$
}

\begin{abstract}
${ }^{1}$ Division of Hospital Medicine, Cincinnati Children's Hospital Medical Center, Cincinnati, Ohio; ${ }^{2}$ Department of Pediatrics, University of Cincinnati College of Medicine, Cincinnati, Ohio; ${ }^{3}$ Children's Hospital Association, Lenexa, Kansas; ${ }^{4}$ Sections of Emergency Medicine and Hospital Medicine, Children's Hospital Colorado, Department of Pediatrics, University of Colorado; 'Division of General Pediatrics, Children's Hospital Boston, Boston, Massachusetts; ' ${ }^{6}$ Department of Pediatrics, Harvard Medical School, Boston, Massachusetts; 'Division of Inpatient Medicine, Primary Children's Hospital, Intermountain Health Care, Salt Lake City, Utah; ${ }^{8}$ Department of Pediatrics, University of Utah, Salt Lake City, Utah; ${ }^{9}$ Institute for Healthcare Delivery Research, Intermountain Healthcare, Salt Lake City, Utah; ${ }^{10}$ Division of Infectious Diseases, Cincinnati Children's Hospital Medical Center, Cincinnati, Ohio.
\end{abstract}

OBJECTIVE: To compare hospital outcomes associated with commonly used antibiotic therapies for aspiration pneumonia in children with neurologic impairment (NI).

DESIGN/METHODS: A retrospective study of children with NI hospitalized with aspiration pneumonia at 39 children's hospitals in the Pediatric Health Information System database. Exposure was empiric antibiotic therapy classified by antimicrobial activity. Outcomes included acute respiratory failure, intensive care unit (ICU) transfer, and hospital length of stay (LOS). Multivariable regression evaluated associations between exposure and outcomes and adjusted for confounders, including medical complexity and acute illness severity.

RESULTS: In the adjusted analysis, children receiving Gram-negative coverage alone had two-fold greater odds of respiratory failure (odds ratio [OR] 2.15; $95 \% \mathrm{Cl}$ : 1.41-3.27), greater odds of ICU transfer (OR 1.80; $95 \%$ $\mathrm{Cl}$ : 1.03-3.14), and longer LOS [adjusted rate ratio (RR)
1.28; $95 \% \mathrm{Cl}: 1.16-1.41]$ than those receiving anaerobic coverage alone. Children receiving anaerobic and Gram-negative coverage had higher odds of respiratory failure (OR $1.65 ; 95 \% \mathrm{Cl}: 1.19-2.28)$ than those receiving anaerobic coverage alone, but ICU transfer (OR 1.15; $95 \% \mathrm{Cl}: 0.73-1.80$ ) and length of stay (RR $1.07 ; 95 \%$ $\mathrm{Cl}: 0.98-1.16)$ did not statistically differ. For children receiving anaerobic, Gram-negative, and P. aeruginosa coverage, LOS was shorter (RR 0.83; 95\% Cl: 0.76-0.90) than those receiving anaerobic coverage alone; odds of respiratory failure and ICU transfer rates did not significantly differ.

CONCLUSIONS: Anaerobic therapy appears to be important in the treatment of aspiration pneumonia in children with NI. While Gram-negative coverage alone was associated with worse outcomes, its addition to anaerobic therapy may not yield improved outcomes. Journal of Hospital Medicine 2020;15:395-402.

(c) 2020 Society of Hospital Medicine
N eurologic impairment (NI) encompasses static and progressive diseases of the central and/or peripheral nervous systems that result in functional and intellectual impairments. ${ }^{1}$ While a variety of neurologic diseases are responsible for $\mathrm{NI}$ (eg, hypoxic-ischemic encephalopathy, muscular dystrophy), consequences of these diseases extend beyond neurologic manifestations. ${ }^{.}$These children are at an increased risk for aspiration of oral and gastric contents given their common comorbidities of dysphagia, gastroesophageal reflux, impaired cough, and respiratory muscle weakness. ${ }^{2}$ While aspiration may manifest as a self-resolving pneumonitis, the presence

"Corresponding Author: Joanna Thomson, MD, MPH;

Email: joanna.thomson@cchmc.org; Telephone: 513-636-0257

Published online first November 20, 2019.

Find additional supporting information in the online version of this article.

Received: May 10, 2019; Revised: September 17, 2019;

Accepted: September 29, 2019

๑ 2019 Society of Hospital Medicine DOI 10.12788/jhm.3338 of oral or enteric bacteria in aspirated material may result in the development of bacterial pneumonia. Children with NI hospitalized with aspiration pneumonia have higher complication rates, longer and costlier hospitalizations, and higher readmission rates when compared with children with nonaspiration pneumonia. ${ }^{3}$

While pediatric aspiration pneumonia is commonly attributed to anaerobic bacteria, this is largely based on extrapolation from epidemiologic studies that were conducted in past decades. ${ }^{4.8} \mathrm{~A}$ single randomized controlled trial found that penicillin and clindamycin, antimicrobials with similar antimicrobial activity against anaerobes, to be equally effective. ${ }^{9}$ However, the recent literature emphasizes the polymicrobial nature of aspiration pneumonia in adults, with the common isolation of Gram-negative enteric bacteria. ${ }^{10}$ Further, while Pseudomonas aeruginosa is often identified in respiratory cultures from children with $\mathrm{Nl}$ and chronic respiratory insufficiency, ${ }^{11,12}$ the significance of $P$. aeruginosa in lower airways remains unclear.

We designed this study to compare hospital outcomes associated with the most commonly prescribed empiric antimicrobial therapies for aspiration pneumonia in children with NI. 


\section{MATERIALS AND METHODS}

\section{Study Design and Data Source}

This multicenter, retrospective cohort study used the Pediatric Health Information System (PHIS) database. PHIS, an administrative database of 50 not-for-profit tertiary care pediatric hospitals, contains data regarding patient demographics, diagnoses and procedures, and daily billed resource utilization, including laboratory and imaging studies. Data quality and reliability are assured through the Children's Hospital Association (CHA; Lenexa, Kansas) and participating hospitals. Due to incomplete data through the study period and data quality issues, six hospitals were excluded.

\section{Study Population}

\section{Inclusion Criteria}

Children 1-18 years of age who were discharged between July 1, 2007 and June 30, 2015 were included if they had a NI diagnosis, ${ }^{1}$ a principal diagnosis indicative of aspiration pneumonia $(507 . x),{ }^{3,13,14}$ and received antibiotics in the first two calendar days of admission. NI was determined using previously defined International Classification of Diseases, Ninth Revision-Clinical Modification (ICD-9-CM) diagnosis codes. ${ }^{1}$ We only included children who received antibiotics in the first two calendar days of admission to minimize the likelihood of including children admitted for other reasons who acquired aspiration pneumonia after hospitalization. For children with multiple hospitalizations, one admission was randomly selected for inclusion to minimize weighting results toward repeat visits.

\section{Exclusion Criteria}

Children transferred from another hospital were excluded as records from their initial presentation, including treatment and outcomes, were not available. We also excluded children with tracheostomy ${ }^{15,16}$ or chronic ventilator dependence, ${ }^{17}$ those with a diagnosis of human immunodeficiency virus or tuberculosis, and children who received chemotherapy during hospitalization given expected differences in etiology, treatment, and outcomes. ${ }^{18}$

\section{Exposure}

The primary exposure was antibiotic therapy received in the first two days of admission. Antibiotics were classified by their antimicrobial spectra of activity as defined by The Sanford Guide to Antimicrobial Therapy ${ }^{19}$ against the most commonly recognized pathogens of aspiration pneumonia: anaerobes, Gram-negatives, and P. aeruginosa (Appendix Table 1). ${ }^{10,20}$ For example, penicillin $G$ and clindamycin were among the antibiotics classified as providing anaerobic coverage alone, whereas ceftriaxone was classified as providing Gram-negative coverage alone and ampicillin-sulbactam or as combination therapy with clindamycin and ceftriaxone were classified as providing anaerobic and Gram-negative coverage. Piperacillin-tazobactam and meropenem were classified as providing anaerobic, Gram-negative, and P. aeruginosa coverage. We excluded antibiotics that do not provide coverage against anaerobes, Gram-negative, or P. aeruginosa (eg, ampicillin, azith- romycin) or that provide coverage against Gram-negative and $P$. aeruginosa, but not anaerobes (eg, cefepime, tobramycin), as these therapies were prescribed for $<5 \%$ of the cohort. We chose not to examine the coverage for Streptococcus pneumonia or Staphylococcus aureus as antibiotics included in this analysis covered these bacteria for $99.9 \%$ of our cohort.

\section{Outcomes}

Outcomes included acute respiratory failure during hospitalization, intensive care unit (ICU) transfer, and hospital length of stay (LOS). Acute respiratory failure during hospitalization was defined as the presence of Clinical Transaction Classification (CTC) or ICD-9 procedure code for noninvasive or invasive mechanical ventilation on day two or later of hospitalization, with or without the need for respiratory support on day 0 or day 1 (Appendix Table 2). Given the variability in hospital policies that may drive ICU admission criteria for complex patients, our outcome of ICU transfer was defined as the requirement for ICU level care on day two or later of hospitalization without ICU admission. Acute respiratory failure and ICU care occurring within the first two hospital days were not classified as outcomes because these early events likely reflect illness severity at presentation rather than outcomes attributable to treatment failure; these were included as markers of severity in the models.

\section{Patient Demographics and Clinical Characteristics}

Demographic and clinical characteristics that might influence antibiotic choice and/or hospital outcomes were assessed. Clinical characteristics included complex chronic conditions, ${ }^{21-23}$ medical technology assistance ${ }_{1}^{24}$ performance of diagnostic testing, and markers of severe illness on presentation. Diagnostic testing included bacterial cultures (blood, respiratory, urine) and chest radiograph performance in the first two days of hospitalization. Results of diagnostic testing are not available in the PHIS. IIIness severity on presentation included acute respiratory failure, pleural drainage, receipt of vasoactive agents, and transfusion of blood products in the first two days of hospitalization (Appendix Table 2). 17,25,26

\section{Statistical Analysis}

Continuous data were described with median and interquartile ranges (IQR) due to nonnormal distribution. Categorical data were described with frequencies and percentages. Patient demographics, clinical characteristics, and hospital outcomes were stratified by empiric antimicrobial coverage and compared using chi-square and Kruskal-Wallis tests as appropriate.

Generalized linear mixed-effects models with random hospital intercepts were derived to assess the independent effect of antimicrobial spectra of activity on outcomes of acute respiratory failure, ICU transfer, and LOS while adjusting for important differences in demographic and clinical characteristics. LOS had a nonnormal distribution. Thus, we used an exponential distribution. Covariates were chosen a priori given the clinical and biological relevance to exposure and outcomes-age, presence of complex chronic condition diagnoses, the number of com- 


\begin{tabular}{|c|c|c|c|c|c|c|c|c|c|c|c|}
\hline \multirow{3}{*}{$\begin{array}{l}\text { Characteristic } \\
\mathrm{N}(\%) \\
\text { Age; median, (IQR) }\end{array}$} & \multirow{2}{*}{\multicolumn{2}{|c|}{$\begin{array}{c}\text { Overall } \\
4,733\end{array}$}} & \multicolumn{2}{|c|}{ Anaerobic } & \multicolumn{2}{|c|}{ Gram-negative $^{\mathrm{a}}$} & \multicolumn{2}{|c|}{$\begin{array}{l}\text { Anaerobic and } \\
\text { Gram-negative }^{\mathrm{a}}\end{array}$} & \multicolumn{2}{|c|}{$\begin{array}{c}\text { Anaerobic } \\
\text { Gram-negative } \\
\text { and } P \text {. aeruginosa }\end{array}$} & \multirow{3}{*}{$\begin{array}{l}\text { PValue }^{\mathrm{b}} \\
<.001\end{array}$} \\
\hline & & & \multirow{2}{*}{$\begin{array}{c}724 \\
5\end{array}$} & \multirow{2}{*}{$\begin{array}{l}(15.3) \\
(2-11)\end{array}$} & \multirow{2}{*}{$\begin{array}{c}503 \\
4\end{array}$} & \multirow{2}{*}{$\begin{array}{l}(10.6) \\
(1-10)\end{array}$} & \multirow{2}{*}{$\begin{array}{c}2,740 \\
5\end{array}$} & \multirow{2}{*}{$\begin{array}{l}(57.9) \\
(2-11)\end{array}$} & \multirow{2}{*}{$\begin{array}{c}766 \\
8\end{array}$} & \multirow{2}{*}{$\begin{array}{l}(16.2) \\
(3-14)\end{array}$} & \\
\hline & 5 & $(2-11)$ & & & & & & & & & \\
\hline Male & 2,553 & $(53.9)$ & 381 & $(52.6)$ & 258 & $(51.3)$ & 1,501 & (54.8) & 413 & $(53.9)$ & .247 \\
\hline Non-Hispanic black & 675 & $(14.3)$ & 131 & $(18.1)$ & 84 & $(16.7)$ & 364 & $(13.3)$ & 96 & $(12.5)$ & \\
\hline Hispanic & 1,213 & (25.6) & 138 & $(19.1)$ & 145 & $(28.8)$ & 751 & (27.4) & 179 & (23.4) & \\
\hline Asian & 166 & (3.5) & 29 & $(4.0)$ & 13 & (2.6) & 101 & $(3.7)$ & 23 & (3.0) & \\
\hline Other & 411 & $(8.7)$ & 63( & 8.7) & 37 & $(7.4)$ & 264 & $(9.6)$ & 47 & $(6.1)$ & \\
\hline Primary source of payment; $n$ (\%) & & & & & & & & & & & .061 \\
\hline \multicolumn{12}{|l|}{ CCC; n (\%) } \\
\hline Neurologic/Neuromuscular & 3,176 & $(67.1)$ & 496 & $(68.5)$ & 299 & $(59.4)$ & 1,821 & (66.5) & 560 & $(73.1)$ & $<.001$ \\
\hline Gastrointestinal & 3,016 & (63.7) & 473 & $(65.3)$ & 295 & $(58.6)$ & 1,705 & $(62.2)$ & 543 & $(70.9)$ & $<.001$ \\
\hline Congenital/Genetic defect & 1,745 & (36.9) & 245 & (33.8) & 202 & $(40.2)$ & 1,008 & (36.8) & 290 & $(37.9)$ & .137 \\
\hline Respiratory & 420 & (8.9) & 56 & $(7.7)$ & 48 & (9.5) & 226 & $(8.2)$ & 90 & $(11.7)$ & .014 \\
\hline Cardiovascular & 638 & $(13.5)$ & 78 & $(10.8)$ & 91 & $(18.1)$ & 353 & $(12.9)$ & 116 & $(15.1)$ & .001 \\
\hline Metabolic & 423 & (8.9) & 72 & $(9.9)$ & 36 & $(7.2)$ & 234 & $(8.5)$ & 81 & $(10.6)$ & .116 \\
\hline Neonatal & 327 & (6.9) & 45 & $(6.2)$ & 37 & $(7.4)$ & 202 & (7.4) & 43 & (5.6) & .307 \\
\hline Renal & 232 & $(4.9)$ & 29 & $(4.0)$ & 19 & (3.8) & 133 & $(4.9)$ & 51 & $(6.7)$ & .053 \\
\hline Malignancy & 129 & $(2.7)$ & 12 & $(1.7)$ & 10 & (2.0) & 56 & $(2.0)$ & 51 & $(6.7)$ & $<.001$ \\
\hline Hematology/Immunodeficiency & 135 & (2.9) & 13 & $(1.8)$ & 19 & (3.8) & 72 & $(2.6)$ & 31 & $(4.0)$ & .030 \\
\hline Gastrointestinal & 2,987 & (63.1) & 468 & (64.6) & 290 & (57.7) & 1,691 & (61.7) & 538 & $(70.2)$ & $<.001$ \\
\hline Respiratory & 5 & $(0.1)$ & 0 & $(00)$ & 0 & (0) & 1 & (0) & 4 & $(0.5)$ & .002 \\
\hline Neurologic/Neuromuscular & 463 & (9.8) & 69 & $(9.5)$ & 55 & $(10.9)$ & 251 & $(9.2)$ & 88 & (11.5) & .209 \\
\hline Cardiovascular & 38 & $(0.8)$ & 2 & $(0.3)$ & 5 & $(1.0)$ & 23 & $(0.8)$ & 8 & $(1.0)$ & .340 \\
\hline Renal & 42 & $(0.9)$ & 3 & $(0.4)$ & 8 & (1.6) & 22 & $(0.8)$ & 9 & $(1.2)$ & .132 \\
\hline Other & 346 & $(7.3)$ & 43 & $(5.9)$ & 39 & $(7.8)$ & 192 & $(7.0)$ & 72 & $(9.4)$ & .059 \\
\hline \multicolumn{12}{|c|}{ Diagnostic testing on presentation; $n$ (\%) } \\
\hline Blood culture & 3,144 & (66.4) & 373 & (51.5) & 333 & $(66.2)$ & 1,841 & $(67.2)$ & 597 & $(77.9)$ & $<.001$ \\
\hline Respiratory viral test & 1,596 & (33.7) & 169 & (23.3) & 173 & (34.4) & 947 & (34.6) & 307 & $(40.1)$ & $<.001$ \\
\hline Respiratory culture & 473 & $(10.0)$ & 39 & $(5.4)$ & 39 & $(7.8)$ & 243 & $(8.9)$ & 152 & $(19.8)$ & $<.001$ \\
\hline Urine culture & 1,284 & $(27.1)$ & 127 & $(17.5)$ & 141 & $(28.0)$ & 710 & (25.9) & 306 & $(39.9)$ & $<.001$ \\
\hline Chest radiograph & 4,283 & $(90.5)$ & 632 & $(87.3)$ & 446 & (88.7) & 2,492 & (90.9) & 713 & (93.1) & $<.001$ \\
\hline Severity of illness on presentation; $n$ & & & & & & & & & & & \\
\hline Acute respiratory failure & 1,201 & (25.4) & 131 & $(18.1)$ & 110 & $(21.9)$ & 642 & (23.4) & 318 & $(41.5)$ & $<.001$ \\
\hline Pleural drainage & 16 & $(0.3)$ & 1 & $(0.1)$ & 2 & $(0.4)$ & 6 & $(0.2)$ & 7 & $(0.9)$ & .022 \\
\hline Receipt of vasoactive agents & 421 & (8.9) & 47 & $(6.5)$ & 57 & $(11.3)$ & 221 & $(8.1)$ & 96 & $(12.5)$ & $<.001$ \\
\hline Transfusion of blood products & 55 & $(1.2)$ & 2 & $(0.3)$ & 7 & (1.4) & 24 & $(0.9)$ & 22 & (2.9) & $<.001$ \\
\hline $\begin{array}{l}{ }^{2} \text { Gram-negative denotes coverage } \\
{ }^{\circ} P \text { value for chi-square test for cat }\end{array}$ & $\begin{array}{l}\text {-nega } \\
\text { les an }\end{array}$ & $\begin{array}{l}\text { thogen } \\
\text { ilcoxon }\end{array}$ & P. a & a & & & & & & & \\
\hline Abbreviations: CCC, chronic cor & QR, & ile & & & & & & & & & \\
\hline
\end{tabular}


TABLE 2. Unadjusted Outcomes

\begin{tabular}{|c|c|c|c|c|c|c|c|c|c|c|c|}
\hline \multirow{2}{*}{$\begin{array}{l}\text { Unadjusted Outcomes }^{\mathbf{a}} \\
\text { Acute respiratory failure }\end{array}$} & \multicolumn{2}{|c|}{ Overall } & \multicolumn{2}{|c|}{ Anaerobic } & \multicolumn{2}{|c|}{ Gram-negative $^{b}$} & \multicolumn{2}{|c|}{$\begin{array}{l}\text { Anaerobic and } \\
\text { Gram-negative }^{\mathrm{b}}\end{array}$} & \multicolumn{2}{|c|}{$\begin{array}{l}\text { Anaerobic } \\
\text { Gram-negative } \\
\text { and } P \text {. aeruginosa }\end{array}$} & \multirow{2}{*}{$\begin{array}{l}\text { PValue }^{\mathrm{c}} \\
<.001\end{array}$} \\
\hline & 1,063 & $(22.5)$ & 101 & (14) & 119 & $(23.7)$ & 579 & $(21.1)$ & 264 & $(34.5)$ & \\
\hline ICU transfer & 181 & (3.8) & 25 & (3.5) & 30 & $(6.0)$ & 109 & $(4.0)$ & 17 & $(2.2)$ & .007 \\
\hline LOS in days (median, [IQR]) & 5 & {$[3,9]$} & 4 & {$[2,7]$} & 7 & {$[3,13]$} & 5 & {$[3,9]$} & 7 & {$[4,12]$} & $<.001$ \\
\hline \multicolumn{12}{|c|}{$\begin{array}{l}\text { Definitions: Acute respiratory failure during hospitalization defined as need for respiratory support on day two or later of hospitalization with or without need for respiratory support on day } 0 \\
\text { day one. ICU transfer defined as requirement for ICU level care on day two or later of hospitalization without ICU admission. } \\
{ }^{\circ} \text { Gram-negative denotes coverage against Gram-negative pathogens except } P \text {. aeruginosa } \\
{ }^{\circ} P \text { value for chi-square test for categorical variables and for Kruskal Wallis test for continuous variables. }\end{array}$} \\
\hline
\end{tabular}

plex chronic conditions, technology dependence, the performance of diagnostic tests on presentation, and illness severity on presentation. ICU admission was included as a covariate in acute respiratory failure and LOS outcome models. The results of the model for acute respiratory failure and ICU transfer are presented as adjusted odds ratios (OR) with a $95 \% \mathrm{Cl}$. LOS results are presented as adjusted rate ratios (RR) with $95 \% \mathrm{Cl}$.

All analyses were performed with SAS 9.3 (SAS Institute, Cary, North Carolina). $P$ values $<.05$ were considered statistically significant. Cincinnati Children's Hospital Medical Center Institutional Review Board considered this deidentified dataset study as not human subjects research.

\section{RESULTS}

\section{Study Cohort}

At the 44 hospitals included, 4,812 children with NI hospitalized with the diagnosis of aspiration pneumonia met the eligibility criteria. However, 79 received antibiotics with the spectra of activity not examined, leaving 4,733 children in our final analysis (Appendix Figure). Demographic and clinical characteristics of the study cohort are shown in Table 1. Median age was five years (interquartile range [IQR]: 2-11 years). Most subjects were male $(53.9 \%)$, non-Hispanic white $(47.9 \%)$, and publicly insured (63.6\%). There was a slight variation in the distribution of admissions across seasons (spring $31.6 \%$, summer $19.2 \%$, fall $21.3 \%$, and winter $27.9 \%$ ). One-third of children had four or more comorbid CCCs (complex chronic conditions; $34.2 \%$ ). The three most common nonneurologic CCC diagnosis categories were gastrointestinal (63.1\%), congenital and/or genetic defects (36.9\%), and respiratory (8.9\%). Assistance with medical technologies was also common (82\%)-particularly gastrointestinal (63.1\%) and neurologic/neuromuscular (9.8\%) technologies. The vast majority of children (92.5\%) had either a chest radiograph $(90.5 \%)$, respiratory viral study $(33.7 \%)$, or respiratory culture (10.0\%) obtained on presentation. A minority required noninvasive or invasive respiratory support (25.4\%), vasoactive agents $(8.9 \%)$, blood products $(1.2 \%)$, or pleural drainage $(0.3 \%)$ in the first two hospital days.

\section{Spectrum of Antimicrobial Coverage}

Most children (57.9\%) received anaerobic and Gram-negative coverage; $16.2 \%$ received anaerobic, Gram-negative and
P. aeruginosa coverage; $15.3 \%$ received anaerobic coverage alone; and $10.6 \%$ received Gram-negative coverage alone. Empiric antimicrobial coverage varied substantially across hospitals: anaerobic coverage was prescribed for $0 \%-44 \%$ of patients; Gram-negative coverage was prescribed for 3\%-26\% of patients; anaerobic and Gram-negative coverage was prescribed for $25 \%-90 \%$ of patients; and anaerobic, Gram-negative, and $P$. aeruginosa coverage was prescribed for $0 \%-65 \%$ of patients (Figure 1).

There were several important differences between treatment groups (Table 1). Children receiving anaerobic, Gram-negative, and $P$. aeruginosa coverage were older, more likely to have certain CCCs (respiratory, gastrointestinal, and malignancy), have $\geq 4$ CCCs, and require assistance with medical technologies (respiratory, gastrointestinal) compared with all other treatment groups. They were also more likely to have respiratory viral testing and bacterial cultures obtained and to have markers of severe illness on presentation.

\section{Outcomes}

\section{Acute Respiratory Failure}

One-quarter (25.4\%) of patients had acute respiratory failure on presentation; $22.5 \%$ required respiratory support (continued from presentation or were new) on day two or later of hospitalization (Table 2). In the adjusted analysis, children receiving Gram-negative coverage alone had two-fold greater odds (OR 2.15, 95\% Cl: 1.41-3.27) and children receiving anaerobic and Gram-negative coverage had 1.6-fold greater odds (OR 1.65, $95 \% \mathrm{Cl}: 1.19-2.28)$, of respiratory failure during hospitalization compared with those receiving anaerobic coverage alone (Figure 2). Odds of respiratory failure during hospitalization did not significantly differ for children receiving anaerobic, Gram-negative, and $P$. aeruginosa coverage compared with those receiving anaerobic coverage alone.

\section{ICU Transfer}

Nearly thirty percent (29.0\%) of children required ICU admission, with an additional 3.8\% requiring ICU transfer following admission (Table 2). In the multivariable analysis, the odds of an ICU transfer were greater for children receiving Gram-negative coverage alone (OR 1.80, 95\% Cl: 1.03-3.14) compared with those receiving anaerobic coverage alone. There was no 


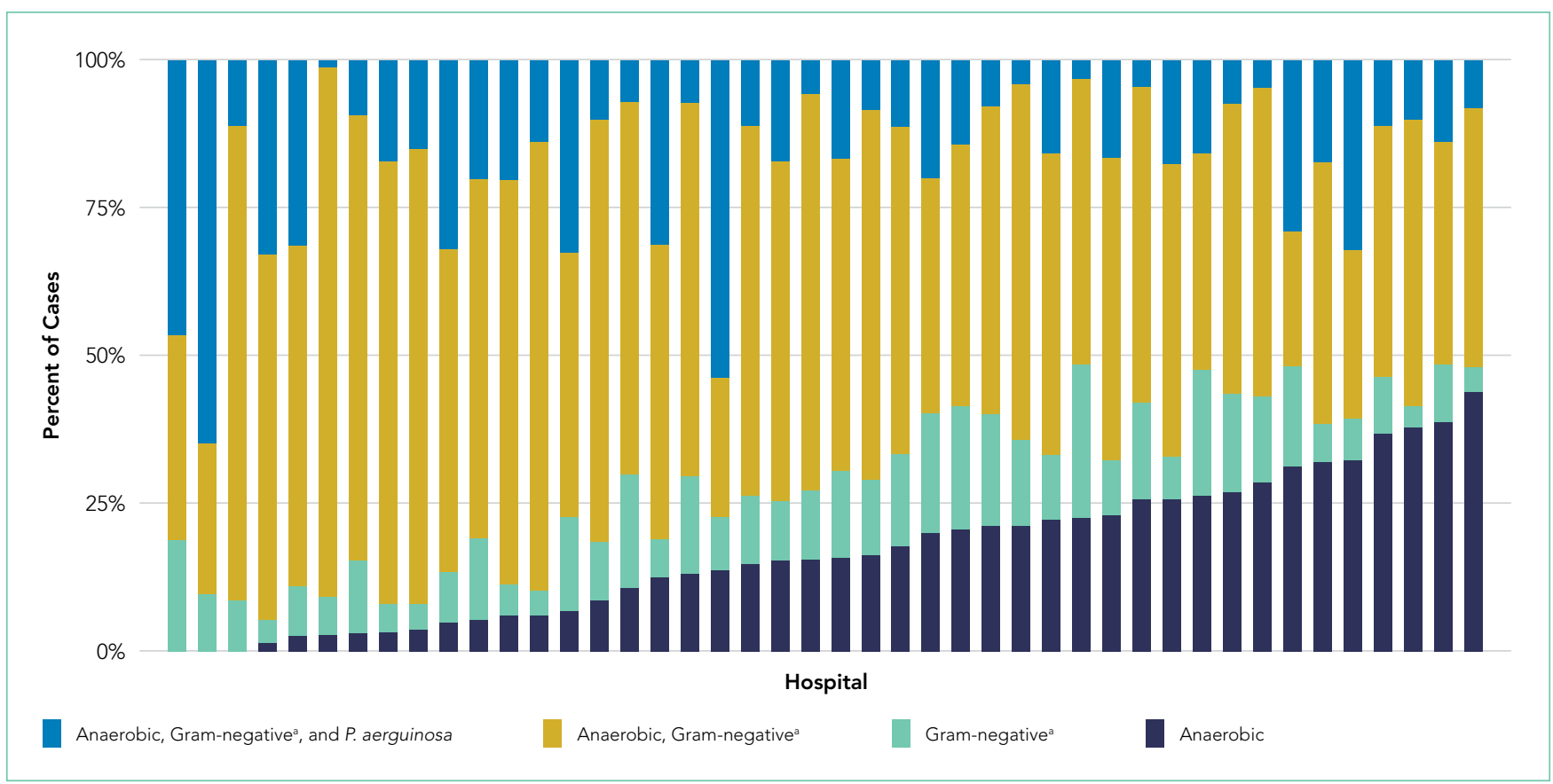

FIG 1. Variation in the Spectrum of Empiric Antimicrobial Coverage across Hospitals.

Each column represents data from one hospital. The spectrum of empiric antimicrobial coverage varied vastly across hospitals: anaerobic coverage was prescribed for $0 \%-44 \%$ of patients; Gram-negative coverage alone was prescribed for 3\%-26\% of patients; anaerobic and Gram-negative coverage was prescribed for $25 \%-90 \%$ of patients; and anaerobic, Gram-negative, and P. aeruginosa coverage was prescribed for $0 \%-65 \%$ of patients.

${ }^{a}$ Gram-negative denotes coverage against Gram-negative pathogens except for $P$. aeruginosa

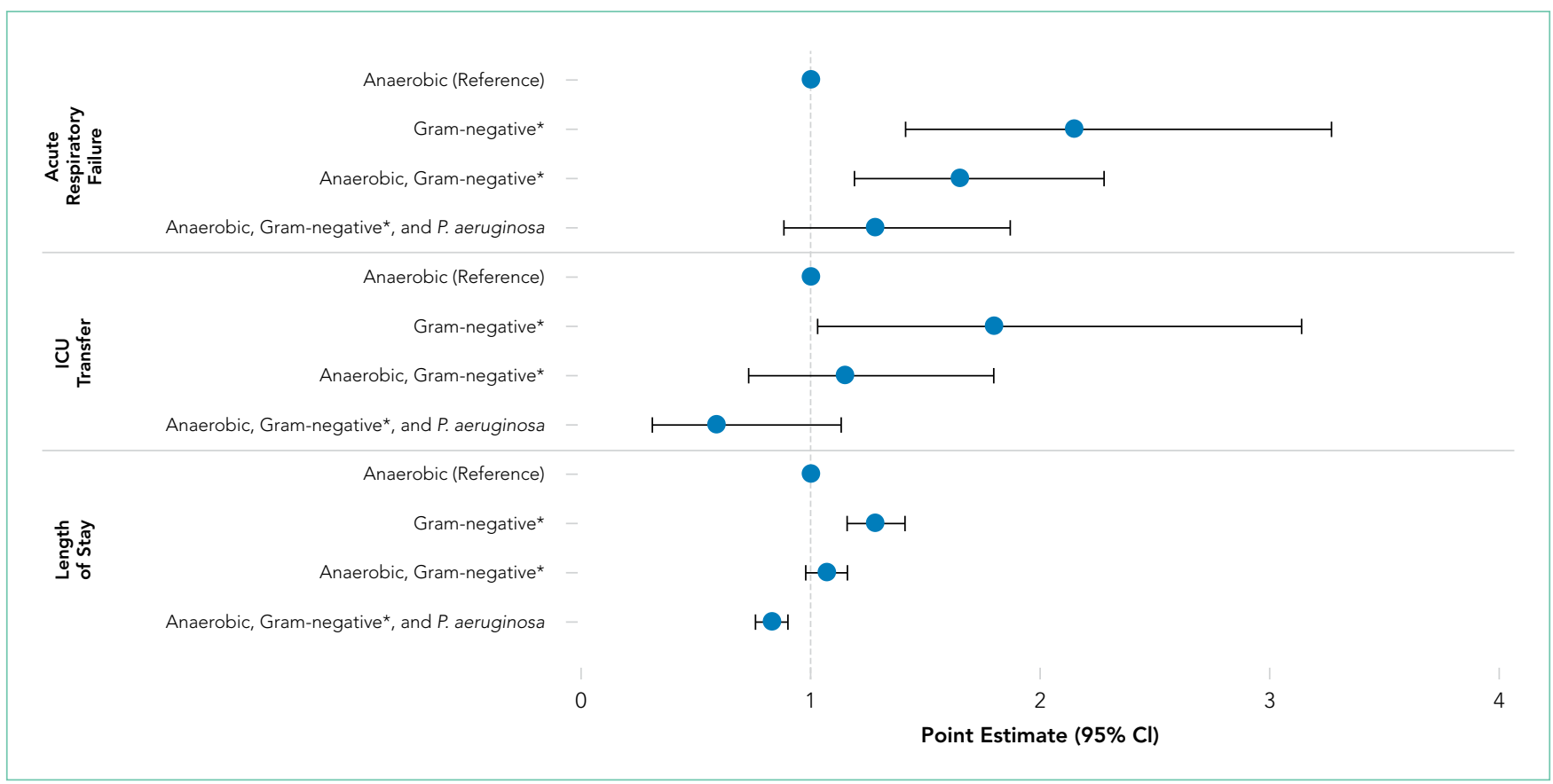

\section{FIG 2. Adjusted outcomes.}

Independent effect of antimicrobial spectra of activity on outcomes (adjusted odds ratio and 95\% $\mathrm{Cl}$ for acute respiratory failure and ICU transfer; adjusted rate ratio for the length of stay) for children with NI hospitalized with aspiration pneumonia. Anaerobic therapy alone served as the referent group. Generalized linear mixed-effects models included hospital as a random effect to account for clustering by hospital and individual covariates (ie, age, presence of complex chronic condition diagnoses (in neurologic/neuromuscular, gastrointestinal, congenital/genetic defect, respiratory, cardiovascular, metabolic, neonatal, renal, malignancy, and hematology/immunology categories), the number of complex chronic conditions, technology dependence (any and gastrointestinal), performance of diagnostic tests on presentation (blood culture, respiratory culture, urine culture, and chest radiograph), and markers of severe illness on presentation (pleural drainage, receipt of blood products, and receipt of vasoactive agents)). ICU admission was included as a covariate in acute respiratory failure and length of stay outcome models.

Abbreviations: ICU, intensive care unit; $\mathrm{NI}$, neurologic impairment. 
statistical difference in ICU transfer for those receiving anaerobic and Gram-negative coverage (with or without $P$. aeruginosa coverage) compared with those receiving anaerobic coverage alone (Figure 2).

\section{Length of Stay}

Median hospital LOS for the total cohort was five days (IQR: 3-9 days; Table 2). In the multivariable analysis, children receiving Gram-negative coverage alone had a longer LOS (RR 1.28; 95\% Cl: 1.16-1.41) compared with those receiving anaerobic coverage alone, whereas children receiving anaerobic, Gram-negative, and $P$. aeruginosa coverage had a shorter LOS (RR 0.83; 95\% Cl: 0.76-0.90) than those receiving anaerobic coverage alone (Figure 2). There was no statistical difference in the LOS between children receiving anaerobic and Gram-negative coverage and those receiving anaerobic coverage alone.

\section{DISCUSSION}

In this multicenter study of children with NI hospitalized with aspiration pneumonia, we found substantial variation in empiric antimicrobial coverage for children with aspiration pneumonia. When comparing outcomes across groups, children who received anaerobic and Gram-negative coverage had outcomes similar to children who received anaerobic therapy alone. However, children who did not receive anaerobic coverage (ie, Gram-negative coverage alone) had worse outcomes, most notably a greater than two-fold increase in the odds of experiencing acute respiratory failure during hospitalization when compared with children receiving anaerobic therapy. These findings support prior literature that has highlighted the importance of anaerobic therapy in the treatment of aspiration pneumonia. The benefit of antibiotics targeting Gram-negative organisms, in addition to anaerobes, remains uncertain.

The variability in empiric antimicrobial coverage likely reflects the paucity of available information on oral and/or enteric bacteria required to identify them as causative organisms in aspiration pneumonia. In part, this problem is due to the difficulty in obtaining adequate sputum for culture from pediatric patients. ${ }^{27}$ While it may be more feasible to obtain tracheal aspirates for respiratory culture in children with a tracheostomy, interpretation of culture results remains challenging because the lower airways of children with tracheostomy are commonly colonized with bacterial pathogens. ${ }^{28}$ Thus, physicians are often left to choose empiric antimicrobial coverage with inadequate supporting evidence. ${ }^{29}$ Although the polymicrobial nature of aspiration pneumonia is well recognized in adult and pediatric literature, ${ }^{10,30}$ it is less clear which organisms are of pathological significance and require treatment.

The treatment standard for aspiration pneumonia has long included anaerobic therapy. ${ }^{29}$ The worse outcomes of children not receiving anaerobic therapy (ie, Gram-negative coverage alone) compared with children who received anaerobic therapy support the continued importance of anaerobic therapy in the treatment of aspiration pneumonia for hospitalized children with NI. The role of antibiotics covering Gram-negative organisms is less clear. Recent studies suggest the role of an- aerobes is overemphasized in the etiology and treatment of aspiration pneumonia. ${ }^{10,29,31-38}$ Multiple studies on aspiration pneumonia bacteriology in hospitalized adults have demonstrated a predominance of Gram-negative organisms (ranging from $37 \%-71 \%$ of isolates identified on respiratory culture) and a relative scarcity of anaerobes (ranging from $0 \%-16 \%$ of isolates). ${ }^{31-37}$ A prospective study of 50 children hospitalized with clinical and radiographic evidence of pneumonia with known aspiration risk (eg, neuromuscular disease or dysphagia) found that $\sim 80 \%$ of 163 bacterial isolates were Gram-negative. ${ }^{38}$ However, this study included repeat cultures from the same children, and thus, may overestimate the prevalence of Gram-negative organisms. In our study, children who received both anaerobic and Gram-negative therapy had no differences in ICU transfer or LOS but did experience higher odds of acute respiratory failure. As these results may be due to unmeasured confounding, future studies should further explore the necessity of Gram-negative coverage in addition to anaerobic coverage in this population.

While these recent studies may seem to suggest that anaerobic coverage is not necessary for aspiration pneumonia, there are important limitations worth noting. First, these studies used a variety of sampling techniques. While organisms grown from samples obtained via bronchoalveolar lavage ${ }^{31-34,36}$ are likely pathogenic, those grown from tracheal or oral samples obtained via percutaneous transtracheal aspiration, ${ }^{34}$ a protected specimen brush, $34,36,37$ or expectorated sputum ${ }^{35,38}$ may not represent lower airway organisms. Second, anaerobic cultures were not obtained in all studies. ${ }^{31,34,38}$ Anaerobic organisms are difficult to isolate using traditional clinical specimen collection techniques and aerobic culture media. ${ }^{18}$ Furthermore, anaerobes are not easily recovered from lung infections after the receipt of antibiotic therapy. ${ }^{39}$ Details regarding pretreatment, which are largely lacking from these studies, are necessary to interpret the relative scarcity of anaerobes on respiratory culture. Finally, caution should be taken when extrapolating the results of studies focused on the etiology and treatment of aspiration pneumonia in elderly adults to children. Our results, particularly in the context of the limitation of these more recent studies, suggest that the role of anaerobes has been underestimated.

Recent studies examining populations of children with cerebral palsy and/or tracheostomy have emphasized the high rates of carriage and infection rates with Gram-negative and drug-resistant bacteria; in particular, P. aeruginosa accounts for $50 \%$ $72 \%$ of pathogenic bacteria. $11,12,38,40$ These studies note the generally poor outcomes of children with $P$. aeruginosa-including multiple and longer hospitalizations, frequent readmissions, and the increased severity of pneumonia, including the need for ICU admission, pleural effusions, the need for intubation, and mortality. ${ }^{11,12,38,40,41}$ In our study, nearly $35 \%$ of children who received anaerobic, Gram-negative, and $P$. aeruginosa coverage experienced acute respiratory failure during hospitalization compared with $20 \%$ of children who received other therapies. While these results might seem to suggest that broader spectrum therapy is harmful, they must be interpreted in the context 
of important population differences; children who received a combination of anaerobic, Gram-negative, and $P$. aeruginosa coverage had greater medical complexity and greater severity of illness on presentation. Such factors may provide the reason for the appropriate prescription of antipseudomonal antibiotics (eg, history of tracheostomy colonization or infection, longterm care facility resident). ${ }^{42}$ When we controlled for population differences, children who received antipseudomonal therapy had a significantly shorter LOS and no differences in outcomes of acute respiratory failure or ICU transfer compared with those receiving anaerobic therapy alone. This result suggests that worse outcomes were associated with antipseudomonal therapy on unadjusted analyses resulting from underlying medical complexity and illness severity rather than from colonization or infection with $P$. aeruginosa.

Our multicenter observational study has several limitations. We used diagnosis codes to identify patients with aspiration pneumonia. As validated clinical criteria for the diagnosis of aspiration pneumonia do not exist, clinicians may assign a diagnosis of and treatment for aspiration pneumonia by subjective suspicion based on a child's severe $\mathrm{NI}$ or illness severity on presentation leading to selection bias. Although administrative data are not able to verify pneumonia type with absolute certainty, we previously demonstrated that the differences in the outcomes of children with aspiration and nonaspiration pneumonia diagnosis codes persist after accounting for the complexity that might influence the diagnosis. ${ }^{3}$ It is also possible that the diagnosis of aspiration pneumonia was not made upon admission for a subset of patients leading to misclassification of exposure. Some children may have had aspiration pneumonia on admission but were not assigned that diagnosis or treated for presumed aspiration pneumonia until later in the hospital course as they demonstrated treatment failure or clinical worsening. It is also possible that some children had an aspiration event during hospitalization that developed into aspiration pneumonia. We attempted to adjust for medical complexity and illness severity through multivariable adjustment based on the diagnosis and procedure codes, as well as the laboratory testing performed. However, unmeasured or residual confounding may remain as administrative data are not equipped to distinguish detailed functional status (eg, ability to cough, chest wall strength) or illness severity (eg, respiratory distress) that might influence antibiotic selection and/or outcomes.

Frthermore, we were unable to account for laboratory, microbiology, or radiology test results, and other management practices (eg, frequency of airway clearance, previous antimicrobial therapy) that may influence outcomes. Future studies should certainly include an examination of the concordance of the antibiotics prescribed with causative organisms, as this undoubtedly affects patient outcomes. Other outcomes are important to examine (eg, time to return to respiratory baseline), but we were unable to do so, given the lack of clinical detail in our database. We randomly selected a single hospitalization for children with multiple admissions; alternative methods could have different results. Although children with
$\mathrm{NI}$ predominately use children's hospitals, ${ }^{1}$ results may not be generalizable.

\section{CONCLUSION}

These findings support prior literature that has highlighted the important role anaerobic therapy plays in the treatment of aspiration pneumonia in children with NI. In light of the limitations of our study design, we believe that rigorous clinical trials comparing anaerobic with anaerobic and Gram-negative therapy are an important and necessary next step to determine the optimal treatment for aspiration pneumonia in this population.

Disclosures: The authors do not have any financial relationships relevant to this article to disclose.

Funding: Dr Thomson was supported by the Agency for Healthcare Research and Quality (AHRQ) under award number K08HS025138. Dr Ambroggio was supported by the National Institute for Allergy and Infectious Diseases (NIAID) under award number K01Al125413. The content is solely the responsibility of the authors and does not necessarily represent the official views of the AHRQ or NIAID.

\section{References}

1. Berry JG, Poduri $A$, Bonkowsky JL, et al. Trends in resource utilization by children with neurological impairment in the United States inpatient health care system: a repeat cross-sectional study. PLoS Med. 2012;9(1):e1001158. https://doi.org/10.1371/journal.pmed.1001158

2. Seddon PC, Khan Y. Respiratory problems in children with neurological impairment. Arch Dis Child. 2003;88(1):75-78. https://doi.org/10.1136/ adc.88.1.75

3. Thomson J, Hall M, Ambroggio L, et al. Aspiration and non-aspiration pneumonia in hospitalized children with neurologic impairment. Pediatrics. 2016;137(2):e20151612. https://doi.org/10.1542/peds.2015-1612

4. Brook I. Anaerobic pulmonary infections in children. Pediatr Emerg Care. 2004;20(9):636-640. https://doi.org/10.1097/01.pec.0000139751.63624.0b

5. Bartlett JG, Gorbach SL. Treatment of aspiration pneumonia and primary lung abscess. Penicillin G vs clindamycin. JAMA. 1975;234(9):935-937. https://doi.org/10.1001/jamadermatol.2017.0297

6. Bartlett JG, Gorbach SL, Finegold SM. The bacteriology of aspiration pneumonia. Am J Med. 1974;56(2):202-207. https://doi.org/10.1016/00029343(74)90598-1

7. Lode H. Microbiological and clinical aspects of aspiration pneumonia. J Antimicrob Chemother. 1988;21:83-90. https://doi.org/10.1093/jac/21.suppl_c.83

8. Brook I. Treatment of aspiration or tracheostomy-associated pneumonia in neurologically impaired children: effect of antimicrobials effective against anaerobic bacteria. Int J Pediatr Otorhinolaryngol. 1996;35(2):171-177. https:// doi.org/10.1016/0165-5876(96)01332-8

9. Jacobson SJ, Griffiths K, Diamond S, et al. A randomized controlled trial of penicillin vs clindamycin for the treatment of aspiration pneumonia in children. Arch Pediatr Adolesc Med. 1997;151(7):701-704. https://doi. org/10.1001/archpedi.1997.02170440063011

10. DiBardino DM, Wunderink RG. Aspiration pneumonia: a review of modern trends. J Crit Care. 2015;30(1):40-48. https://doi.org/10.1016/j. jcrc.2014.07.011

11. Gerdung CA, Tsang A, Yasseen AS, 3rd, Armstrong K, McMillan HJ, Kovesi T. Association between chronic aspiration and chronic airway infection with Pseudomonas aeruginosa and other Gram-negative bacteria in children with cerebral palsy. Lung. 2016;194(2):307-314. https://doi.org/10.1007/s00408-016-9856-5

12. Thorburn K, Jardine M, Taylor N, Reilly N, Sarginson RE, van Saene HK. Antibiotic-resistant bacteria and infection in children with cerebral palsy requiring mechanical ventilation. Pedr Crit Care Med. 2009;10(2):222-226. https:// doi.org/10.1097/PCC.0b013e31819368ac

13. Lanspa MJ, Jones BE, Brown SM, Dean NC. Mortality, morbidity, and disease severity of patients with aspiration pneumonia. J Hosp Med. 2013;8(2):83-90. https://doi.org/10.1002/jhm.1996

14. Lanspa MJ, Peyrani P, Wiemken T, Wilson EL, Ramirez JA, Dean NC. Characteristics associated with clinician diagnosis of aspiration pneumonia: a descriptive study of afflicted patients and their outcomes. J Hosp Med. 
2015;10(2):90-96. https://doi.org/10.1002/jhm.2280

15. Berry JG, Graham RJ, Roberson DW, et al. Patient characteristics associated with in-hospital mortality in children following tracheotomy. Arch Dis Child. 2010;95(9):703-710.

16. Berry JG, Graham DA, Graham RJ, et al. Predictors of clinical outcomes and hospital resource use of children after tracheotomy. Pediatrics. 2009;124(2):563-572. https://doi.org/10.1136/adc.2009.180836

17. Balamuth $F$, Weiss $S L$, Hall M, et al. Identifying pediatric severe sepsis and septic shock: Accuracy of diagnosis codes. J Pediatr. 2015;167(6):1295-1300 e1294. https://doi.org/10.1016/j.jpeds.2015.09.027

18. American Academy of Pediatrics., Pickering LK, American Academy of Pe diatrics. Committee on Infectious Diseases. In: Red book : 2012 report of the Committee on Infectious Diseases. 29th ed. Elk Grove Village: American Academy of Pediatrics; 2012.

19. Gilbert DN. The Sanford Guide to Antimicrobial Therapy 2014. 44th ed Sperryville: Antimicrobial Therapy, Inc; 2011.

20. Marik PE. Aspiration pneumonitis and aspiration pneumonia. N Engl J Med. 2001;344(9):665-671. https://doi.org/10.1056/NEJM200103013440908

21. Feudtner C, Feinstein JA, Zhong W, Hall M, Dai D. Pediatric complex chronic conditions classification system version 2: updated for ICD-10 and complex medical technology dependence and transplantation. BMC Pediatrics. 2014;14:199. https://doi.org/10.1186/1471-2431-14-199

22. Feudtner C, Hays RM, Haynes G, Geyer JR, Neff JM, Koepsell TD. Deaths attributed to pediatric complex chronic conditions: national trends and implications for supportive care services. Pediatrics. 2001;107(6):E99. https:// doi.org/10.1542/peds.107.6.e99

23. Feinstein JA, Russell S, DeWitt PE, Feudtner C, Dai D, Bennett TD. R pack age for pediatric complex chronic condition classification. JAMA Pediatr. 2018;172(6):596-598. https://doi.org/10.1001/jamapediatrics.2018.0256

24. Berry JG, Hall DE, Kuo DZ, Cohen E, Agrawal R, Feudtner C, Hall M, Kueser J, Kaplan W, Neff J. Hospital utilization and characteristics of patients experiencing recurrent readmissions within children's hospitals. JAMA. 2011;305(7):682-690. https://doi.org/10.1001/jama.2011.122

25. Shah SS, Hall M, Newland JG, et al. Comparative effectiveness of pleura drainage procedures for the treatment of complicated pneumonia in childhood. J Hosp Med. 2011;6(5):256-263. https://doi.org/10.1002/jhm.872

26. Child Health Corporation of America. СTC ${ }^{\mathrm{TM}} 2010$ Code Structure: Module 5 Clinical Services. 2010 January 4. https://sharepoint.chca.com/CHCAForums/Performancelmprovement/PHIS/Reference Library/CTC Resources/ Forms/Allltems.aspx Version: Modified

27. Bradley JS, Byington CL, Shah SS, et al. The management of community-acquired pneumonia in infants and children older than 3 months of age: clinical practice guidelines by the Pediatric Infectious Diseases Society and the Infectious Diseases Society of America. Clin Infect Dis. 2011;53(7):e25-76. https://doi.org/10.1093/cid/cir531

28. Brook I. Bacterial colonization, tracheobronchitis, and pneumonia following tracheostomy and long-term intubation in pediatric patients. Chest $1979 ; 76(4): 420-424$
29. Waybright RA, Coolidge W, Johnson TJ. Treatment of clinical aspiration: a reappraisal. Am J Health Syst Pharm. 2013;70(15):1291-1300. https://doi. org/10.2146/ajhp120319

30. Brook I, Finegold SM. Bacteriology of aspiration pneumonia in children. Pediatrics. 1980;65(6):1115-1120.

31. Wei C, Cheng Z, Zhang L, Yang J. Microbiology and prognostic factors of hospital- and community-acquired aspiration pneumonia in respiratory intensive care unit. Am J Infect Control. 2013;41(10):880-884. https://doi. org/10.1016/j.ajic.2013.01.007

32. El-Solh AA, Pietrantoni $C$, Bhat A, et al. Microbiology of severe aspiration pneumonia in institutionalized elderly. Am J Respir Crit Care Med. 2003;167(12):1650-1654. https://doi.org/10.1164/rccm.200212-15430C

33. Tokuyasu H, Harada T, Watanabe $E$, et al. Effectiveness of meropenem for the treatment of aspiration pneumonia in elderly patients. Intern Med. 2009;48(3):129-135. https://doi.org/10.2169/internalmedicine.48.1308

34. Ott SR, Allewelt M, Lorenz J, Reimnitz P, Lode H, German Lung Abscess Study Group. Moxifloxacin vs ampicillin/sulbactam in aspiration pneumonia and primary lung abscess. Infection. 2008;36(1):23-30. https://doi.org/10.1007/ s15010-007-7043-6

35. Kadowaki M, Demura Y, Mizuno S, et al. Reappraisal of clindamycin IV monotherapy for treatment of mild-to-moderate aspiration pneumonia in elderly patients. Chest. 2005;127(4):1276-1282. https://doi.org/10.1016/j.chest.2017.05.019

36. Marik PE, Careau P. The role of anaerobes in patients with ventilator-associated pneumonia and aspiration pneumonia: a prospective study. Chest. 1999;115(1):178-183. https://doi.org/10.1378/chest.115.1.178

37. Mier L, Dreyfuss D, Darchy B, et al. Is penicillin $G$ an adequate initial treatment for aspiration pneumonia? A prospective evaluation using a protected specimen brush and quantitative cultures. Intensive Care Med. 1993;19(5):279284. https://doi.org/10.1007/bf01690548

38. Ashkenazi-Hoffnung L, Ari A, Bilavsky E, Scheuerman O, Amir J, Prais D. Pseudomonas aeruginosa identified as a key pathogen in hospitalised children with aspiration pneumonia and a high aspiration risk. Acta Paediatr. 2016;105(12):e588-e592. https://doi.org/10.1111/apa.13523

39. Bartlett JG, Gorbach SL, Tally FP, Finegold SM. Bacteriology and treatment of primary lung abscess. Am Rev Respir Dis. 1974;109(5):510-518. https://doi. org/10.1164/arrd.1974.109.5.510

40. Russell CJ, Simon TD, Mamey MR, Newth CJL, Neely MN. Pseudomonas aeruginosa and post-tracheotomy bacterial respiratory tract infection readmissions. Pediatr Pulmonol. 2017;52(9):1212-1218. https://doi.org/10.1002/ ppul.23716

41. Russell CJ, Mamey MR, Koh JY, Schrager SM, Neely MN, Wu S. Length of stay and hospital revisit after bacterial tracheostomy-associated respiratory tract infection hospitalizations. Hosp Pediatr. Hosp Pediatr. 2018;8(2):72-80. https://doi.org/10.1542/hpeds.2017-0106

42. Russell CJ, Mack WJ, Schrager SM, Wu S. Care variations and outcomes for children hospitalized with bacterial tracheostomy-associated respiratory infections. Hosp Pediatr. 2017;7(1):16-23. https://doi.org/10.1542/ hpeds.2016-0104 